\title{
Evaluation of admissions to the Major Incident Hospital based on a standardized protocol
}

\author{
G. M. H. Marres • J. van der Eijk • \\ M. Bemelman • L. P. H. Leenen
}

Received: 3 July 2010/ Accepted: 19 November 2010/Published online: 3 February 2011

(C) The Author(s) 2011. This article is published with open access at Springerlink.com

\begin{abstract}
Introduction The Major Incident Hospital (MIH) is a unique facility strictly reserved to provide immediate largescale emergency care for victims of disasters and major incidents. We evaluated the implemented organization to identify strengths and weaknesses, and provide knowledge essential for further improvement of preparedness.

Method According to the Protocol for Reports from Major accidents and Disasters (PRMD) and along with our five scenarios for activation, we analyzed all the data from evaluation reports of all our deployments since the MIH was founded in 1991.

Results The MIH was able to provide group-wise emergency care to military (29 admissions) as well as civilian victims of major incidents and disasters, both national (260) and international (226). Group-wise treatment was advantageous for quarantine, logistics, registration, emotional support and (pre)arrangements for family, media and security. Strong points are preparedness and availability of a dedicated facility, including ICU, X-ray and OR facilities, irrespective of MRSA status and prearranged cooperation, e.g., with a trauma centre, poison centre and the military. Evaluation, research and training resulted in a
\end{abstract}

Electronic supplementary material The online version of this article (doi:10.1007/s00068-010-0067-0) contains supplementary material, which is available to authorized users.

G. M. H. Marres - J. van der Eijk - M. Bemelman ·

L. P. H. Leenen

Department of Surgery and Trauma, University Medical Centre

Utrecht and Central Military Hospital, Utrecht, The Netherlands

G. M. H. Marres $(\bowtie)$

Department of Surgery and Trauma, University Medical Centre Utrecht, Heidelberglaan 100, 3584 CX Utrecht, The Netherlands e-mail: g.m.h.marres@umcutrecht.nl barcode registration system and continuous adaptations to improve preparedness. Shortage of resources did not occur; use of the MIH's available resources for national incidents though, could be further optimized.

Conclusions Recommendations for the future are: improvement of imbedding in regional and national procedures, continued dedicated time and staff for training, research and development, improvement of nuclear/biological/chemical decontamination facilities and preparedness, implementation of standardized scoring systems and expansion of registration systems to the prehospital setting.

Keywords Disaster medicine - Evaluation study . Dormant major incident hospital · Disaster preparedness . Major incidents
Abbreviations
CMH Central Military Hospital
EAN European Article Numbering
ICU Intensive Care Unit
MIH Major Incident Hospital
MRSA Methicillin-resistant Staphylococcus aureus
NVIC National Poison Information Centre
PBRS Patient Barcode Registration System
PRMD Protocol for Reports from Major Accidents and Disasters
SARS Severe Acute Respiratory Syndrome, caused by the SARS coronavirus
UMCU University Medical Centre Utrecht

\section{Introduction}

A disaster or major incident causes a sudden, unexpected surge of casualties. In general, disasters produce physical 
injury. Medical management does not simply involve treating these like in regular trauma care in upscaled proportions, but requires a paradigm change in concepts, approaches and mindset [1]. Preparation and training is essential to cope with the challenge of providing optimal care and to minimize excess morbidity and mortality [1,2].

Since 1991, the Netherlands has had a Major Incident Hospital (MIH) to provide immediate medical emergency care for multiple casualties under exceptional circumstances. This unique facility is strictly reserved, with expertise and complete infrastructure waiting to provide large scale emergency care with disasters and major incidents [3].

Its missions are divided into five scenarios indicating deployment:

1. War (threat), crisis or conflict management in which large numbers of casualties need care

2. Accidents abroad involving Dutch citizens, civilian or military, in need of repatriation and medical care

3. Specific incidents, attacks or large-scale accidents in the Netherlands that exceed the regular care capacity

4. International medical assistance from the Dutch government concerning the treatment of foreign victims

5. Quarantine care for patients with special infectious and highly contagious diseases, such as SARS (Severe Acute Respiratory Syndrome).

The opening of the MIH is an essential part of the disaster plan of the University Medical Centre Utrecht (UMCU) and the Central Military Hospital (CMH). The $\mathrm{MIH}$ is located in a nuclear bunker under the UMCU, which is in turn connected to the CMH by a corridor. An Emergency Response Protocol enables admittance to the normally dormant hospital of up to 100 patients after a start-up time of only $15 \mathrm{~min}$. With an additional $45 \mathrm{~min}$ the patient count can be extended to 300 . Personnel are alerted through a personnel alert system. The organization, infrastructure and training are all directed around triage to guide patient flow through successive echelons of care in order to deliver the greatest care to the greatest number of people (Figs. 1, 2). The Patient Barcode Registration System (PBRS) with European Article Numbering (EAN) codes guarantees quick and adequate registration to facilitate good medical coordination and follow-up during a major incident [4].

As analysis of the implemented organization and clinical management could identify helpful decisions and errors, we evaluated the use of the MIH and its admissions during the past 19 years. Research and evaluation of past incidents provide knowledge for preparedness and response to unknown future incidents and are essential for further improvements [5]. Systematically evaluating the quality of medical response to major incidents is difficult, though,

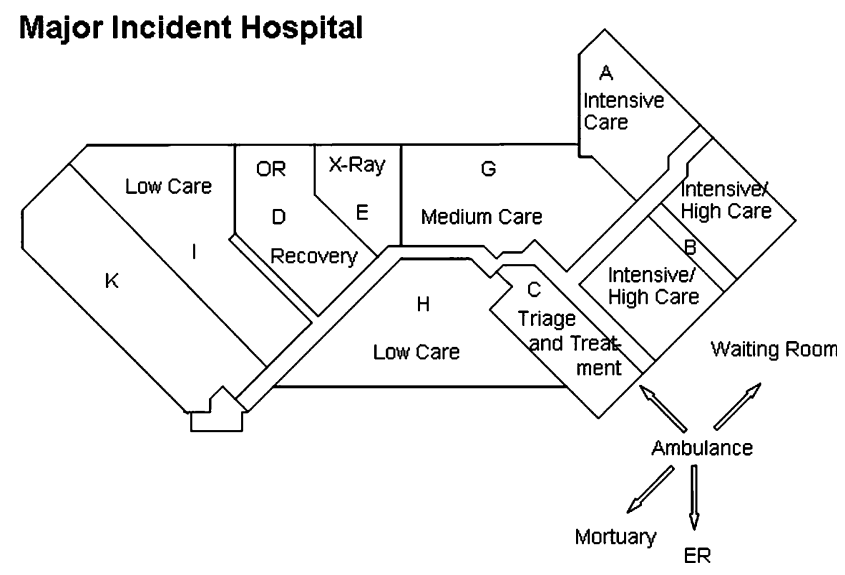

Fig. 1 Map of the MIH

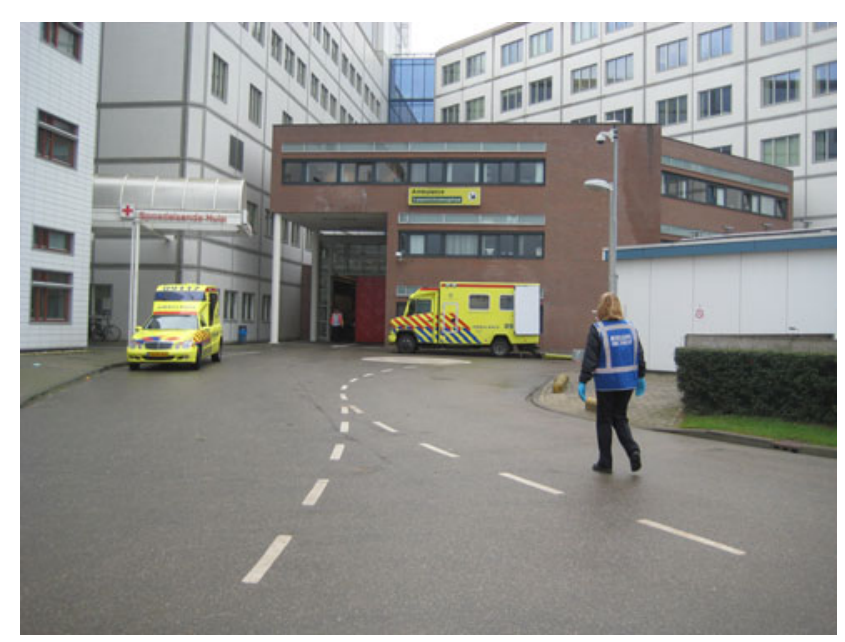

Fig. 2 Entrance of the ambulance hall of the MIH

especially because of obvious differences in the types of disasters and a wide variety in modes of evaluation. Furthermore, there is no standardization of reporting yet. Performance indicators for the quality of the medical response are scarce but have been evolving in the past decade. Critical mortality rate (the percentage of severely wounded victims who die), for example, gives an indication of the magnitude of the disaster and of the effectiveness of the medical management $[5,6]$. To evaluate the performance of the MIH, however, this tool would be too general and more reflective of the performance of the total of disaster response than of hospital care alone. Ruter et al. [7] suggest a set of 20 performance indicators that they applied to 13 earlier reports on major incidents issued by the KAMEDO organization in Sweden. These consist mainly of timeframes for decisions and actions at the scene and at the level of command and control, predominantly prehospital. They also developed some performance indicators for testing the skills of hospital management groups in simulation exercises [8]. Some indicators of preparedness, not 
performance, have been used to evaluate US trauma centre preparedness [9]. The Utstein template [10] also suggests a method for a more scientific approach. For this goal, it seems too elaborate and theoretical. Lennquist suggested a template to report major incidents, the Protocol for Reports from Major accidents and Disasters (PRMD) [11]. It offers a standardized methodology for reporting results and experiences from major accidents and disasters to improve comparability. It facilitates exchange of experiences and promotes international collaboration in methodological development. The protocol is divided in 18 headings concerning components of the incident as well as of the response, complemented with standardized tables. Although originally developed to evaluate and report about individual major incidents, it offers us a concise and practical template to direct the evaluation of the MIH deployment, as well as a standardized method for reporting.

\section{Objective}

In this study, we evaluate the use of the MIH since its founding in 1991, according to the PRMD [11] and along with our five scenarios for activation. Further central questions were: how did we perform in the five openings scenarios? What are the strong points in group treatment? Which changes led to identifiable and sustainable improvements? Was the MIH effectively used in the whole chain of disaster management? What are our recommendations for the future?

\section{Materials and methods}

Our evaluation concerns the performance of the MIH itself. Inclusion of the prehospital evaluation for 34 incidents is beyond the scope of this analysis.

We retrieved all data from the evaluation reports of every MIH deployment since its founding in 1991. Reports had been made directly after the event as part of the debriefing in almost every admission, and had been discussed among involved disciplines for learning points and accuracy. One of the authors (JvdE) participated in all admissions and evaluations until 2008 except the repatriation of fugitives from Kosovo (1999). Missing data were retrieved retrospectively from the charts and medical files. These files had all been kept on paper within the MIH.

Demographic and quantitative data were registered (such as number of patients, types of injuries, hospital resources). All applicable data were registered according to the PMRD protocol [11], which is divided into 18 headings that we used to direct our evaluation (see Table 1). The standardized tables from the protocol were used,
Table 1 Headings from protocol for reports from major accidents and disasters [10]

\begin{tabular}{ll}
\hline & Headings \\
\hline 1 & Short summary of the scenario* \\
2 & Description of hazard(s) causing the accident* \\
3 & Description of the accident* \\
4 & Prehospital resources available and alerted \\
5 & Hospital resources available and alerted* \\
6 & Utilization of transport resources \\
7 & Hospital alert plan and response* \\
8 & Coordination and command* \\
9 & Hospital damage* \\
10 & Communication system* \\
11 & Computer Technology and back-up systems* \\
12 & Total number and type of injuries* \\
13 & Severity of injuries* \\
14 & Hospital load* \\
15 & Psychological reactions* \\
16 & Outcome \\
17 & Estimated number of people affected but not injured \\
18 & Post-accident evaluation* \\
\hline
\end{tabular}

* Data available for analysis

sometimes extended with additional columns. For qualitative, descriptive values we abstracted the results of all admissions and further discussed admissions which revealed the most interesting learning points.

\section{Results}

The subheadings in this paragraph are analogous to the 18 subheadings of the PRMD.

1/2/3 Summary of the scenario/hazard, description of accident

All 34 consecutive deployments of the MIH are depicted in Table 2, summarized with short descriptions of each event and the type of activation scenario. Military victims (29) accounted for $5 \%$ of the admitted patients and $18 \%$ of the deployments. Two hundred and twenty-six (38\%) Dutch victims of major incidents abroad were repatriated in 12 group admissions, whereas national incidents accounted for 260 patients (47\%) and $35 \%$ of the deployments of the MIH. The rest consist of 4 openings to offer care for foreign incident disaster victims (44 patients, 10\%). Special infectious disease admissions did not take place. For national incidents the distances from the scene to the MIH $(0.3-60 \mathrm{~km})$ are also depicted in Table 2. For international deployment, 
Table 2 Overview of use of the MIH: year, number of admitted patients, short description of the incident, distance (km) from the scene, cohort quarantine and main type of scenario for deployment of the MIH (see below)

\begin{tabular}{|c|c|c|c|c|c|}
\hline Year & $\begin{array}{l}\text { Number } \\
\text { of } \\
\text { patients }\end{array}$ & Incident characteristics (location of incident) & $\begin{array}{l}\text { Distance, or } \\
\text { country if } \\
\text { abroad }\end{array}$ & Quarantine & $\begin{array}{l}\text { Main } \\
\text { scenario* }\end{array}$ \\
\hline 1991 & 9 & Methyl bromide intoxication (used by gardeners decontaminating greenhouses) & $45 \mathrm{~km}$ & No & 3 \\
\hline 1991 & 9 & Carbon monoxide intoxication; insufficient boiler in non ventilated space (Utrecht) & $5-10 \mathrm{~km}$ & No & 3 \\
\hline 1992 & 10 & Carbon monoxide intoxication of fireman on duty & $5-10 \mathrm{~km}$ & No & 3 \\
\hline 1992 & 5 & Exposure to nitrous damps $(\ldots)$ & $5-10 \mathrm{~km}$ & No & 3 \\
\hline 1992 & 28 & Evacuation of a hospital in a war setting (Bosnia Herzegovina) & Bosnia & Yes & 4 \\
\hline 1992 & 13 & Plane crash (Faro) with a fire & Portugal & Yes & 2 \\
\hline 1993 & 43 & Bus accident (Lyon) & France & Yes & 2 \\
\hline 1993 & 5 & Evacuation of a hospital in a war setting (Bosnia Herzegovina) & Bosnia & Yes & 4 \\
\hline 1994 & 4 & Bus accident (Regensburg, Germany) & Germany & Yes & 2 \\
\hline 1995 & 143 & Evacuation of a flooded Hospital & $52 \mathrm{~km}$ & No & 3 \\
\hline 1995 & 7 & Benzyl bromide exposure (Utrecht) & $5-10 \mathrm{~km}$ & No & 3 \\
\hline 1996 & 29 & Bus accident of Dutch singing choir (Winterberg) & Germany & Yes & 2 \\
\hline 1996 & 12 & Cannabis intoxication (Zeist) & $8 \mathrm{~km}$ & No & 3 \\
\hline 1996 & 6 & Aluminium pollution of fluids in dialysis centre & Curacao & No & 4 \\
\hline 1996 & 11 & Bus accident (Antalya) & Turkey & Yes & 2 \\
\hline 1996 & 15 & Carbon monoxide intoxication by inadequate boiler in insufficiently ventilated building & $5-10 \mathrm{~km}$ & No & 3 \\
\hline 1996 & 10 & Bus accident of tourist bus from Netherlands (Thionville) & France & Yes & 2 \\
\hline 1997 & 7 & Military accident (Novi Travnik, Bosnia Herzegovina) & Bosnia & Yes & 1 \\
\hline 1997 & 13 & Bus accident & Syria & Yes & 2 \\
\hline 1998 & 20 & Bus accident of tourist bus from the Netherlands (Metz) & France & Yes & 2 \\
\hline 1999 & 5 & Repatriation of fugitives & Kososvo & Yes & 4 \\
\hline 1999 & 12 & Earthquake, Turkish, Dutch residents on holiday & Turkey & Yes & 2 \\
\hline 2000 & 9 & Military accident & Germany & No & 1 \\
\hline 2002 & 25 & Bus accident of tourist bus from Netherlands (Metz, France) & France & Yes & 2 \\
\hline 2002 & 21 & Bus accident of tourist bus from Netherlands (Manching, Germany) & Germany & Yes & 2 \\
\hline 2003 & 13 & Lab accident, analysis of glue-damp with eye and airway irritation (methyl metacrylate) & $0.3 \mathrm{~km}$ & No & 3 \\
\hline 2005 & 25 & Repatriation of wounded Dutch tsunami victims & Asia & Yes & 2 \\
\hline 2007 & 6 & Collapse of crane on a campus building & $0.4 \mathrm{~km}$ & No & 3 \\
\hline 2007 & 4 & Repatriation of soldiers from Afghanistan (victims of a suicide bombing while on patrol) & Afghanistan & Yes & 1 \\
\hline 2008 & 25 & $\begin{array}{l}\text { Evacuation ( } 350 \text { emloyees) from a contaminated building in Amersfoort (unknown } \\
\text { substance) }\end{array}$ & $23 \mathrm{~km}$ & No & 3 \\
\hline 2009 & 6 & Airplane crash near Schiphol airport ("Polder Crash") with 135 passengers & $60 \mathrm{~km}$ & No & 3 \\
\hline 2009 & 4 & Repatriation of military victims Afghanistan & Afghanistan & Yes & 1 \\
\hline 2009 & 2 & Repatriation of military victims Afghanistan & Afghanistan & Yes & 1 \\
\hline 2009 & 3 & Repatriation of military victims Afghanistan & Afghanistan & Yes & 1 \\
\hline Total & 559 & & & & \\
\hline
\end{tabular}

* Scenario's for activation of the MIH: (1) Military victims, (2) repatriation of Dutch victims of accidents or incidents abroad, cohort-isolation for Methicilline Resistant Staphylococcus Aureus (MRSA) according to guidelines of the Netherlands Health Care Inspectorate, (3) activation of the MIH for a national Major Incident or disaster, (4) international medical assistance from the Dutch government concerning the treatment of foreign victims, (5) quarantine care for patients with special infectious and highly contagious diseases, e.g. SARS

the country of repatriation is depicted, at distances varying from several hundred to thousands of kilometers.

4. Evaluation of "Prehospital resources available and alerted" lies beyond the scope of this report

\section{Hospital resources available or alerted}

As the MIH is a reserved facility for major incidents, availability of triage rooms (35 beds), low and medium care beds (200), ICU beds (12), ventilators, radiology unit, operating theaters and trauma unit is 
guaranteed. Usability is dependent on the ability to staff them. In all evaluated deployments, staffing has never proved to be a problem; the capacity could always be operational for the first 100 beds within $15 \mathrm{~min}$ to half an hour. Training issues of deployed staff are discussed below (Figs. 3, 4, 5, 6).

In the evaluation of optimal use of resources, situations in which the MIH was available but not deployed are particularly interesting.

An example is the Volendam café fire on New Year's Eve, 2001, where 98 victims were transported to 20 hospitals and 100 victims went to different ERs of hospitals on their own. Four people died. The MIH was alerted $7 \mathrm{~h}$ after the incident with the request to receive two burn patients that could be regularly admitted to the UMCU. In total 11 patients were admitted to the UMCU. Numerous patients were transferred to a widespread variety of hospitals and it took a full day afterwards to get an overview. A bus

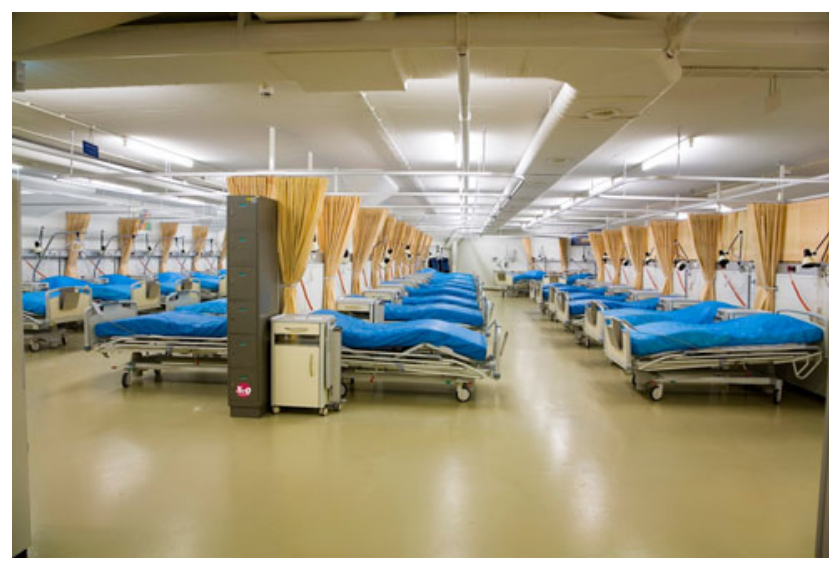

Fig. 3 Low care unit in dormant times

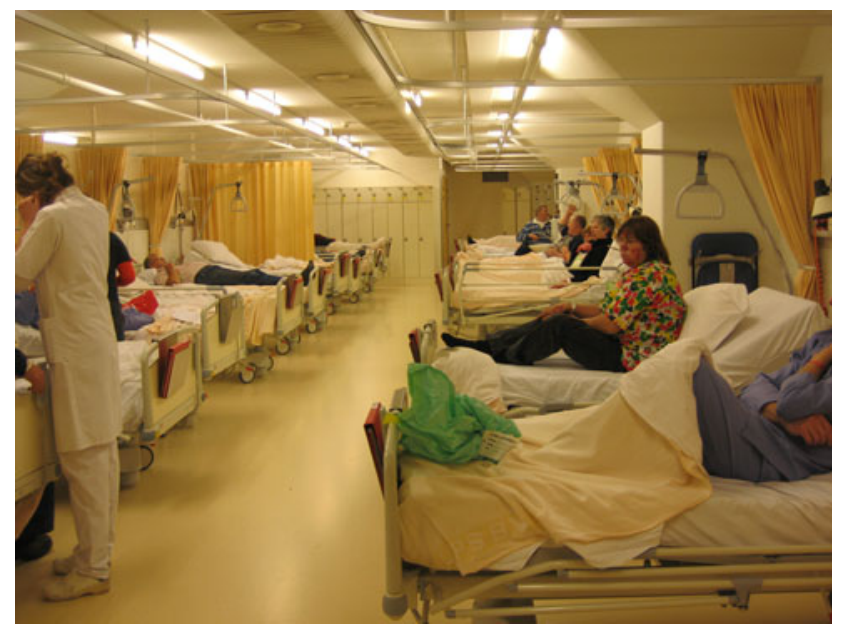

Fig. 4 Low care unit in use

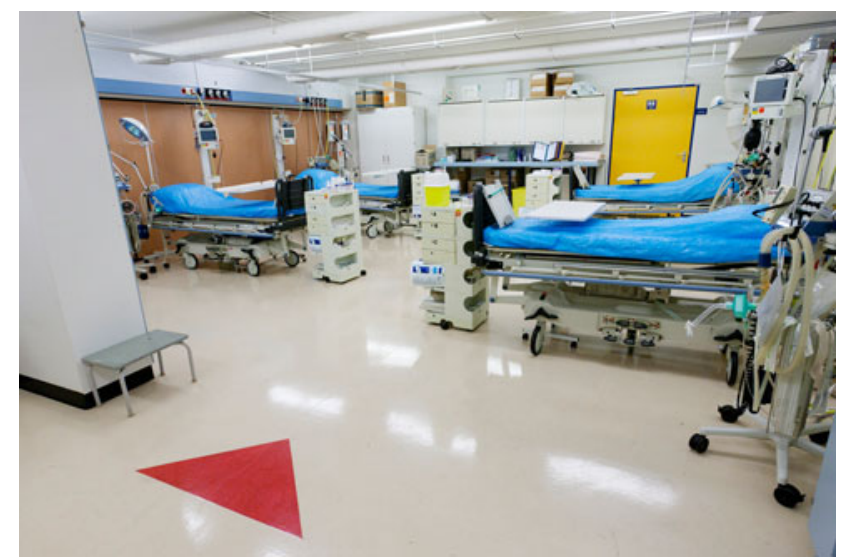

Fig. 5 Triage and treatment area, red bay (photo B de Meijer)

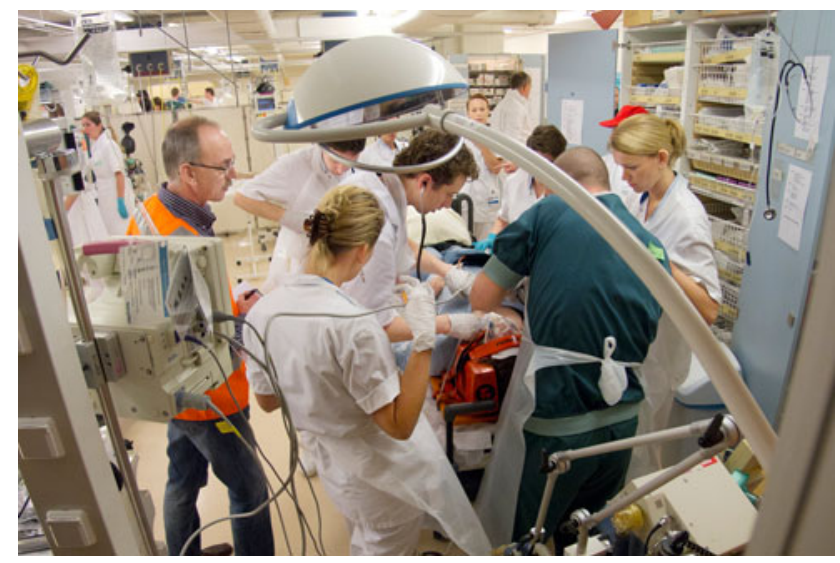

Fig. 6 Triage and treatment area-red bay, stabilisation of a patient (photo B de Meijer)

with $20 \mathrm{~T} 3$ victims (in the four scale triage system [12]) was sent to a hospital that turned out to have no capacity left and had to divert to another hospital [13]. The MIH could have better contributed to care and simplifying patient logistics had it been deployed in an earlier stage.

The collapse of stairs during a musical parade in 2008 [14] is an example where 19 patients were seen in one trauma centre and the MIH was not deployed. Medical management went well and was within surge capacity of the hospital. In this case, using the trauma centre or MIH were both good options and the choice was a matter of preference of the staff on call. Using the MIH for first relief would have eased logistics and communication with supporting facilities that were now problematic due to provisional patient numbering [14]. After the plane crash near Schiphol ("Polder Crash" 2009), 42 of the 135 victims patients were transported from the triage site by bus to a sports complex after being triaged as $\mathrm{T} 3$. The two triage doctors at the sports 
complex concluded that they needed hospital transfer due to high energy characteristics of the crash and triage levels higher than T3. They were spread to several hospitals and it took 4 days to reconstruct the victim list and whereabouts of the patients [15]. Only six patients were transported to the MIH.

One of the other specialties of the MIH is relief of groups of patients in quarantine, which otherwise often generates logistic problems and delay of repatriation to regular hospitals.

In 1994, a group of Dutch victims from a bus accident in Poland were repatriated to a city hospital instead of to the MIH due to political choices of the hometown government, despite prior alert and readiness of the MIH. This resulted in a forced temporary closure of the regular surgery and orthopedic departments due to MRSA contamination.

In the Faro plane crash in 1992, 95 patients were repatriated to 6 hospitals including the MIH. Eight (8\%) proved to be infected with MRSA. This corresponds with $14 \%$ of the MRSA category II patients (patients with minimum $24 \mathrm{~h}$ stay or invasive procedures in a foreign hospital). This led to secondary spread to other patients and staff in one of the Burn Centers.

Repatriation in quarantine from Thailand to the MIH after the 2004 tsunami showed several carriers of MRSA and other multi-resistant microorganisms [16]. Repatriations of military victims from Kosovo and Afghanistan to our facility also showed multiple victims with MRSA and Acinetobacter colonization.

Of all the quarantine admissions, $61 \%$ or more of the patients could be discharged home within the 5 days that are set as a directive to close the MIH, or quarantine could be ended due to negative swabs. Transfers under quarantine conditions were reduced to $32 \%$ of the patients who were initially treated in quarantine.

During the SARS threat we adapted our facilities and training to prepare for patients with special highly infectious diseases. Since 2003, the MIH has changed the air treatment and can provide 4 isolation boxes and 30 medium care beds for this type of scenario.

6. Utilization of transport resources was not applicable/ evaluated.

7. Hospital alert plan and response

Opening of the MIH is always based on activation of the local disaster plan. When the Emergency Response Program is started, the medical manager of the Emergency Room activates the personnel alert system through the national civilian telephone network. This system has specially reserved capacity for use by the disaster management chain. During all deployments, communication through this system was never hindered by the inundation of the regular mobile network, as often occurs in disaster situations. In contrast to a normal, time consuming "calling tree", results are monitored and adjustable once running, as personnel respond to the system if and when they are available. This system enabled us to tailor the alert. At first, we worked with different lists adapted to several scenarios but these incurred too much overlap. In the current system, we can compose the alerted staff by choosing and combining groups, e.g., "base", "surgery", "anesthesia" etc. The system is tested every 6 months for effectivity and to keep numbers updated. Alerts resulted in an average direct availability of $50 \%$, with slightly lower rates at night. Under-alert has not occurred, except for two cases were the radiology department was not included in the initial call. Over-alert was the case in the Polder Crash (2009). Within 45 min after the crash we contacted the regional command center dealing with the crash to inform them that we were available to receive patients within half an hour. This was after seeing the information on the Internet and after the UMCU had been alarmed by the regional ambulance service. In fact, the MIH was never formally approached or informed by the central coordination centre nor by the national crisis team. The MIH was opened based on controversial information from the scene and a second alert from the regional ambulance service that the first four patients were already on their way to our centre. One hundred forty-three staff members were alerted, of which 39 were deployed and the rest were sent home. Finally, only six patients (four T2 and two T3) were transferred to our hospital [15].

8. Organization of coordination and command

Evaluation of preparedness and function scores of all prehospital units of the response chain lies beyond the scope of this report.

For the MIH, the trauma surgeon on call is fully licensed to open the MIH, which benefitted opening speed. Hereafter, a dual command system is implemented. This means that the Command Team focuses on organization of the relief and treatment of the victims in the Major Incident Hospital itself, and a separate Management Team is responsible for external logistics, continuity of the care in the $\mathrm{CMH}$ and UMC, internal and external communication and the organization of first relief of family/relatives. This proved to keep the Command Team alert and in control and prevented distraction by external business beyond patient relief.

9. Hospital damage due to a disaster was never the case. Admission of 13 patients with an inhalation problem from the UMCU laboratory and the collapse of the crane on the same university campus were the nearest scenarios. 


\section{Communication system}

In the beginning, team leaders carried portable phones. This proved distracting as it generated a lot of (unnecessary) use and people did not always know how to use them effectively, not being accustomed to them in the daily setting. We abandoned them (apart from ambulance hall) and chose regular pagers. This resulted in strict reserved use and effectivity. After 1992 the PBRS provided continuous and updated group and patient data in every area and at every working station in the MIH. This resulted in clear improvement in medical, nursing and logistic handling and covered many of the communication needs. It enabled staff to better anticipate and manage care requirements and effectively use resources and personnel up to discharge [17]. Short "chief meetings" took place at regular intervals. During these meetings, the operational unit supervisors discuss all crucial steps with the command team and make logistic decisions and other arrangements between all participating units, ranging from security to operation rooms. The supervisors return to their units to implement the decisions. This proved very effective in sharing information and plans quickly and greatly simplified procedures to get things done and manage the entire organisation.

11. Computer technology and back-up systems

We did not encounter major computer system failures during admissions. Regular exercises in the MIH helped us to improve ease of use. A backup system is present but has never been used. The PBRS that we developed showed $25 \%$ fewer inaccuracies when compared with the handwritten medical charts, despite the greater number of items entered [17]. Extensive training proved to be unnecessary. (Para-) medical personnel judged the automated procedures an improvement. The coupling with the Hospital Information system worked well. The follow up chart of patient care in the MIH is not computerized, yet; that is a goal for the future.

12. Total number and type of injuries

Total number and type of injuries are depicted in Table 3. Almost half of the patients $(254,45 \%)$ were admitted with injuries due to mechanical violence. Inhalation accounts for $16 \%$ (93) of the admitted patients.

13. Severity of injuries

All patients were triaged on admission. Except for triage on admission, we did not use a standard scoring system as for example, the Injury Severity Score (ISS). Severity of injuries according to
Table 3 Total number and type of injuries

\begin{tabular}{lc}
\hline Type & Number of injured \\
\hline Mechanical violence & 254 \\
Military (29) & \\
Non-military (225) & 13 \\
Fire & 93 \\
Inhalation & \\
Methyl bromide (9) & \\
Carbon monoxide (34) & \\
Nitrous damps (5) & \\
Other (45) & 0 \\
Corrosive agents & 0 \\
Irradiation & 0 \\
Cold & 0 \\
Drowning & 0 \\
Biological contamination & 199 \\
Other & \\
Mixed hospital population, external evacuation causes \\
(war, flooding) (181) \\
Cannabis intoxication (12) \\
Aluminium intoxication (6) \\
Total \\
\hline
\end{tabular}

treatment is depicted in Table 4 and was relatively low (as well as retrospectively estimated ISS above 15). Most patients ( $>95 \%)$ were triaged at priority 2 or 3 . Intensive care use $(4 / 559=0.7 \%)$ and ventilator dependency were scarce $(4 / 559=0.7 \%)$, as was need of immediate surgery (defined as within $1 \mathrm{~h})(0 \%)$.

\section{Hospital load}

Congestion, lack of material, resources or personnel influencing optimal patient care did not occur in any of the deployments. Volunteers from the Red Cross to assist in patient care and transport within the MIH proved to be a valuable addition to the professional pool (Fig. 7). Discharge and transfer procedures were delayed and sometimes congested because often many patients were discharged at the same time and definite discharge papers had to be prepared, which requires staff time. Therefore, in 1999, we developed an automated patient discharge letter system and appointed one person in medical staff to supervise this. This improved the discharge process, resulting in no more delays.

15. Psychological reactions and management

In every admission, staff members from the psychology and psychiatric department are available to provide psychological support for victims, relatives and staff. They are supported by volunteers from the Red Cross who assist patients in personal care and 
Table 4 Severity of injuries according to treatment

\begin{tabular}{|c|c|c|c|}
\hline \multirow[t]{2}{*}{ Type } & \multicolumn{3}{|l|}{ Number } \\
\hline & Repatriation & $\begin{array}{l}\text { National } \\
\text { incident }\end{array}$ & Total \\
\hline $\begin{array}{l}\text { Less severe injuries not examined or } \\
\text { treated by medical professionals }{ }^{\mathrm{a}}\end{array}$ & & & n.a. \\
\hline $\begin{array}{l}\text { Less to moderately severe injuries } \\
\text { examined and/or treated by medical } \\
\text { professionals }^{\mathrm{a}}\end{array}$ & & & \\
\hline Outside hospital & & & n.a. \\
\hline In hospital & 2 & 13 & 15 \\
\hline $\begin{array}{l}\text { Injuries requiring in-hospital care/ } \\
\text { observation in }\end{array}$ & 297 & 247 & 544 \\
\hline Regular ward & 289 & 175 & 464 \\
\hline Medium care & 4 & 72 & 76 \\
\hline Intensive care & 4 & 0 & 4 \\
\hline Requiring ventilator & 4 & 0 & 4 \\
\hline $\begin{array}{l}\text { Injuries requiring surgery (general } \\
\text { anaesthesia) }\end{array}$ & $31^{*}$ & $0^{*}$ & $31 *$ \\
\hline Immediately & 0 & 0 & 0 \\
\hline Within $6 \mathrm{~h}$ & 7 & 0 & 7 \\
\hline Delayed & $24 *$ & $0^{*}$ & $24 *$ \\
\hline
\end{tabular}

n.a. not applicable

* In four admissions several additional delayed surgery procedures in referral clinic

${ }^{a}$ Medical professionals defined as ambulance staff, paramedics, nurses and doctors

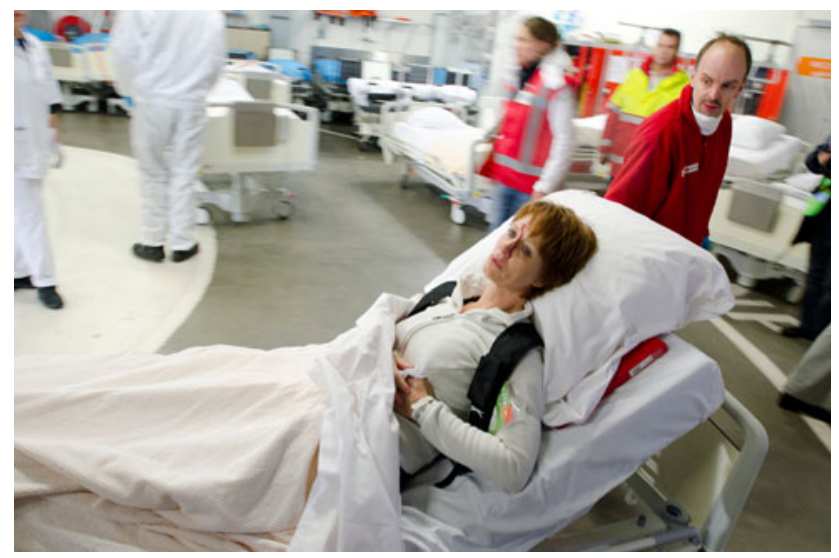

Fig. 7 Patient transport by volunteers of the Red Cross (photo B de Meijer)

offer a listening ear. If desired, the team can provide referral advice for support after discharge. Patients rate this as very positive in patient evaluations. Being among peers was perceived to be supportive and contributing to resilience. Long term evaluation among the repatriated tsunami victims showed that need for professional support often arises at a later stage than directly after the disaster, sometimes even after months or years [18]. We are currently exploring how we can address this by offering a gateway to professional help through a site that can be accessed at any time after the disaster. In analogy with the site launched after the tsunami (http://www.tisei.org) [19] this site combines self help through mutual victim contact and self-assessment with eConsult possibilities.

Post-deployment evaluations with personnel showed that they experienced substantially more emotional burden when caring for incident victims than in regular hospital care. This was not determined by severity of wounds or physical trauma but mostly by the impressive stories, emotional reunion of victims with families and (media) attention that comes with major incidents. During victim relief and during shift changes, a consultative psychiatric nurse is present for personnel. An aftercare team contacts them afterwards. Informal gatherings after the admission proved to give more room for sharing emotions and feelings than meetings labeled to address emotional impact.

16. Outcome with regard to mortality and persistent disability

In-hospital mortality was low $(1 / 559=0.2 \%)$. This concerns a patient that was repatriated from a military mission to confirm brain death and to stop treatment amidst his family. Persistent disability was not scored and was difficult to quantify, as the MIH is a short-term facility that closes after a few days. Opening time ranged between 1 and 5 days. Clinical patients were then transferred to other hospitals and follow-up of outpatients was done mainly in the home city hospitals. From the 559 patients, 256 patients (46\%) were transferred to other hospitals, while 303 (54\%) could be discharged home within closing time of the MIH.

17. Estimated number of people affected, not injured was not scored.

18. Post-accident evaluation

Post accident evaluation of preparedness

The disaster plan is the basis for the MIH and was always activated in the case of admissions. The evaluations showed that the basic disaster plan functioned well. Some of the adaptations are described in the above paragraphs. Another important finding in the evaluations concerns preparedness for situations that require decontamination. During the evacuation from a building contaminated with an initially unknown gas in 2008, patients entered the MIH without decontamination. Afterwards, decontamination proved unnecessary as it turned out to be smoke from an aircon fire, but this reinforced 


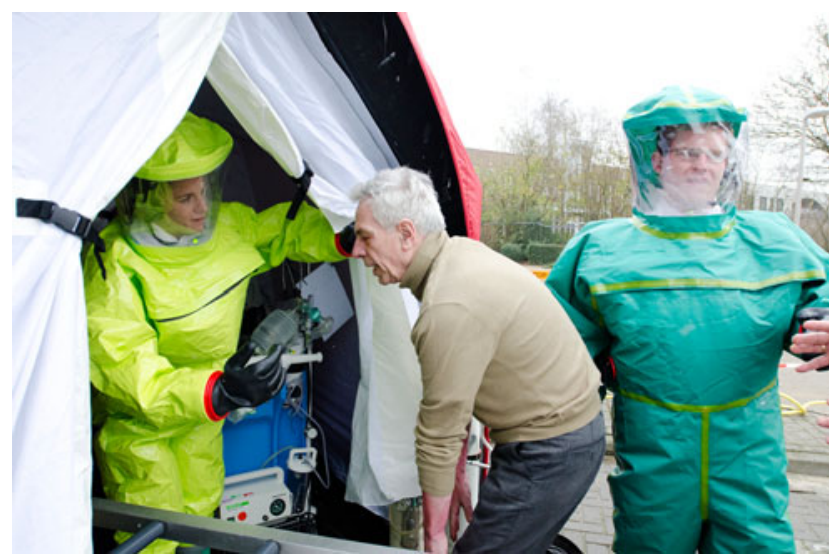

Fig. 8 Training with decontamination in a multidisciplinary excercise with 100 victims, MIH 2010

reevaluation of the disaster plans regarding when and how to start decontamination procedures. In a training session for decontamination within the MIH, we also concluded that our ability to decontaminate large groups of patients was insufficient. We further extended training and are currently improving the infrastructure and decontamination facilities (Fig. 8). Post accident evaluation of training In the first years, optimal relief was sometimes hindered by the fact that the majority of the alarmed personnel was still relatively unfamiliar with procedures and logistics in the MIH. This mandated the expansion of training activities and starting up a pool of personnel who spend part of their time performing tasks in regular care when the MIH is dormant, but spend the rest of their time outside deployment times on training and preparedness. They now form the basis for training the rest of the pool of dedicated doctors, nurses and support team from all UMCU/CMH departments that can be alerted. Regular theoretical classes, case exercises and disaster simulations within the MIH or together with the whole chain resulted in smoother cooperation and better preparedness (Fig. 9). In an environment where teams are always assembled from a big pool, and of which a considerable portion is made up of residents that change every 2 years, the intensity of training with a strong base proved crucial.

\section{Discussion}

The goal of availability, preparedness and quick performance was met in four of the five deployment criteria of the MIH. A major incident with contagious diseases or material (fifth criterion, added since 2003) has not yet occurred.

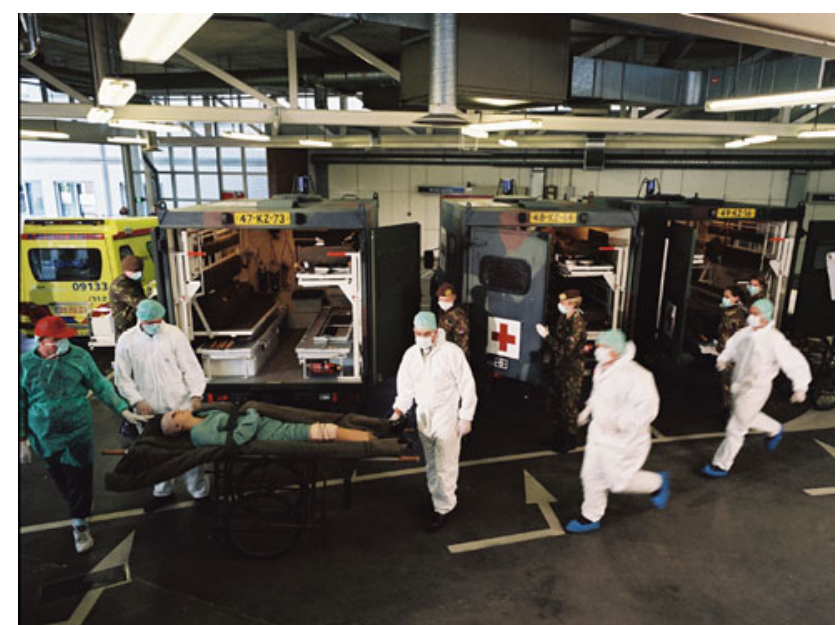

Fig. 9 Cooperation between military and civil services (Ambulance hall $\mathrm{MIH})$

In the relief and repatriation of military victims (first scenario) the MIH has proved its surplus value in availability of the total range of care (low care till ICU and OR as well as radiology facilities) without quarantine restrictions and disturbance of regular care. This facilitates quick repatriation and initiation of treatment, which is often not possible in war/mission setting. MIH staff is partly military and is familiar with repatriation procedures and concurrent emotional impact for patients and family. The same advantages account for civil repatriations (second scenario) in quarantine after incidents and disasters abroad. The Netherlands follows a strict quarantine policy for all foreign patients in order to rule out potential infection with MRSA and other multi-resistant bacteria to prevent transmission to other patients or healthcare workers. This policy is reinforced by recent literature that shows that MRSA types have a predominantly regional distribution in Europe [20]. This finding is indicative of the selection and spread of a limited number of clones within health care networks, suggesting that control efforts aimed at interrupting the spread within and between health care institutions may not only be feasible but ultimately successful and, therefore, desirable [20]. In this evaluation, (MRSA) quarantine has again proved necessary in several deployments and nondeployments. Some examples are discussed above. Groupwise relief in quarantine has the advantage of availability, risk reduction for regular care and reduction of further quarantine efforts. In the quarantine admissions, the majority of the patients could be discharged home before closure of $\mathrm{MIH}$, or quarantine could be ended due to negative swabs. Transfers under quarantine conditions could be greatly reduced and have the advantage of preparation time for the receiving hospital. Preparation time is not only favorable for quarantine issues but time also permitted selection of the most suitable follow-up hospital 
for each individual patient with regard to expertise and geography. Since the repatriation from the bus accident in Poland (1994), the Dutch policy is to perform all group wise repatriations in quarantine in the MIH.

For the third opening scenario, national major incidents, the deployment of the MIH was relatively low and its availability and capacity were certainly not used to maximum advantage. Distance to the scene might be a reason for using other facilities, but in many cases this was not an issue. Utrecht and the MIH are located quite centrally in the Netherlands. For example, in the Polder Crash the MIH was well within $60 \mathrm{~km}$ of the accident, comparable to distances to some of the other deployed hospitals. Direct availability for the entire group would have eased logistics.

We believe the most important factor is suboptimal embedding of the MIH in the Dutch system of incident relief. The organization of disaster relief in the Netherlands is strongly developing and new liaisons with the $\mathrm{MIH}$ are still in progress. The fact that the MIH is not used in regular trauma care might also contribute to suboptimal use of its capacity. The regional medical officer in charge of an incident, as well as the regional command center, might not always be familiar with the advantages the MIH has to offer. The National Coordination centre was not always deployed, although it is appointed for interregional activities and major incidents. Furthermore, hospitals in the affected region, and especially trauma centers, are eager to care for "their own" patients and do what they trained for in trauma care and in their hospital disaster plans. Performance of the regular Dutch trauma centers and hospitals proved to be good for multiple trauma/incident patients in terms of direct care within surge capacity $[14,15,21]$. Leap-frogging could guard these capacities in not-tooextensive incidents, but it also complicates communication, patient overview, logistics, impact on regular care (overand under-alert of several hospitals), family support, media and security $[13,15,21]$. The MIH could make a valuable contribution in effective use of all available capacity and delivering the best care to the greatest number of patients. This does not necessarily mean that all patients should always go to the MIH. A good distribution could be to transport T1 victims to trauma centers and group T2 and T3 victims to the MIH. This allows trauma centers and local hospitals to focus on T1 victims and unavoidable selfreferrals. Meanwhile the MIH can sort out the bulk, eventually with some of the T1 victims, in a situation that is designed for further triage through successive echelons of care, and where other conditions for good group wise treatment are prearranged, such as reserved availability of resources (100-300 beds; 12 ICU, 38 medium care and 50 low care, OR, radiology, etc.), registration, psychological support, media, family issues and security. This could facilitate prehospital organization and quicker patient overview [15]. In contrast to the situation after the Polder Crash, for example, where completing the victim list took 4 days, institutions have only to contact one hospital with regard to numbers of patients, severity of injury and personal data. The PBRS directly provides a real time overview. Relief for T1 victims is also possible in the MIH, but this patient group benefits most from fast treatment, justifying transportation to the nearest adequate facility. Furthermore, they generally have a longer and more intense treatment path that merits significant and highly qualified personnel and resources. Spreading of these patients among trauma centers can probably guarantee better continuity of care after first relief. For these patients the additional value of being among fellow survivors is less relevant.

Irrespective of the type of scenario, group-wise treatment had several advantages, including mutual support among patients and (pre)arrangements for logistics, registration, family and media.

Looking at the other points we evaluated, communication and computer technologies deserve further development. Although the PBRS functions well and brings unique advantages for real time overview and management, extension of patient registration and tracking to the prehospital setting would be desirable. For this goal we developed a Victim Tracking and Tracing System (ViTTS), that will be discussed in another paper. Digitalization of the entire medical chart within the MIH would maximize yield of the PBRS, the discharge letter system and would facilitate research.

Furthermore, we aim to implement the ISS [22] for registration of injury severity. This is an internationally used scoring system, useful for categorizing severity as well as for reporting. This would allow easier comparisons, also with regular trauma care and with outcome related to ISS. Among our admitted patients were relatively few T1 patients or patients with retrospectively estimated injury severity scores above 15. This observation has three explanations: In some incidents severely injured patients went to other hospitals (e.g., Polder Crash 2009), in some cases (e.g., crane collapse, bus accident and evacuation of flooded hospital) the incident itself produced mainly $\mathrm{T} 2$ or $\mathrm{T} 3$ victims, as in most mechanical disasters [23] and finally because some admissions were repatriations with a selected group that was stable enough to travel (e.g., tsunami repatriation).

\section{Conclusions}

The MIH has demonstrated its ability to provide group-wise emergency care to military as well as civil victims of major incidents and disasters, national or from abroad, in all scenarios for activation except care for special highly infectious diseases (as this did not occur). Group-wise treatment 
proved to be advantageous for quarantine, logistics, registration, emotional support and (pre)arrangements for family, media and security. Preparedness and availability, irrespective of MRSA status, including ICU, X-ray and OR facilities and prearranged cooperation, e.g., with the UMCU, NVIC and the military, are strong points. Evaluation and training resulted in developments such as the PBRS and continuous adaptations to improve preparedness.

Key recommendations for further development are:

- Better imbedding in regional and national procedures to optimize use of availability in case of national disasters; expand involvement of other trauma centers.

- Continued training, research and development, preservation of a pool of dedicated staff to keep the organization alive and moving.

- Improvement of nuclear/biological/chemical decontamination facilities, adaptation to external developments.

- Expansion of the PBRS within the MIH and in the prehospital phase: create fully electronic patient charts and prehospital patient tracking and tracing.

- Implementation of standardized scoring systems to record injury type and severity to facilitate research and comparability.

Conflicts of interest We have no conflict of interest to the subject matters discussed in the manuscript.

Open Access This article is distributed under the terms of the Creative Commons Attribution Noncommercial License which permits any noncommercial use, distribution, and reproduction in any medium, provided the original author(s) and source are credited.

\section{References}

1. Frykberg ER. Disaster and mass casualty management: a commentary on the American College of Surgeons position statement. J Am Coll Surg. 2003;197(5):857-9.

2. Lennquist S. Management of major accidents and disasters: an important responsibility for the trauma surgeons. J Trauma. 2007;62(6):1321-9.

3. Marres GMH, Bemelman M, van der Eijk J, Leenen LPH. Major incident hospital: development of a permanent facility for management of incident casualties. Eur J Trauma Emerg Surg. 2009;35(3):203-11.

4. Bouman JH, Schouwerwou RJ, Van der Eijk KJ, van Leusden AJ, Savelkoul TJ. Computerization of patient tracking and tracing during mass casualty incidents. Eur J Emerg Med Sep. 2000;7(3):211-6.

5. Frykberg ER. Medical management of disasters and mass casualties from terrorist bombings: How can we cope? J Trauma Aug. 2002;53(2):201-12.

6. Frykberg E. (2005) Triage: the key to success in mass casualty disasters. Post graduate course 15. Disaster and Mass Casualty Managment. San Francisco: American College of Surgeons; 2005. p. 39-41.
7. Ruter A, Ortenwall P, Wikstrom T. Performance indicators for major incident medical management, a possible tool for quality control? International Journal of Disaster Medicine. 2004;2(1): $52-5$.

8. Ruter A, Nilsson H, Vilkstrom T. Performance indicators as quality control for testing and evaluating hospital management groups: A pilot study. Prehosp Disaster Med. 2006;21(6):423-6.

9. Trunkey D. US trauma center preparation for a terrorist attack in the community. Eur J Trauma Emerg Surg. 2009;35(3):244-64.

10. Sundnes KO. Health disaster management: Guidelines for evaluation and research in the Utstein style: Executive summary Task force on quality control of disaster management. Prehosp Disaster Med. 1999;14(2):43-52.

11. Lennquist S. Protocol for reports from major accidents and disasters in the international journal of disaster medicine. Eur $\mathrm{J}$ Trauma Emerg Surg. 2008;34(5):486-92.

12. Baker MS. Creating order from chaos. Part I. Triage, initial care, and tactical considerations in mass casualty and disaster response. Mil Med Mar. 2007;172(3):232-6.

13. Ministry of Health, Welfare and Sport. Evaluation of medical relief in new years eve cafe fire (rapport evaluatie geneeskundige hulpverlening cafebrand nieuwjaarsnacht 2001). Leiden, The Netherlands: de Bink; 2001.

14. Bemelman M, Leenen L. Mass casualty event during a musical parade: Lessons learned. Eur J Trauma Emerg Surg. 2008; 34(5):451-6.

15. Ministry of the Interior and Kingdom relations, Public Order and Safety Directorate. Poldercrash February 25th 2009. A report from the public order and safety directorate. (poldercrash 25 februari 2009. Een onderzoek door de inspectie van openbare orde en veiligheid, in samenwerking met de inspectie voor de gezondheidszorg). Den Haag, The Netherlands: Public Order and Safety Directorate; 2009.

16. Marres GMH, de Lange D, Leenen LPH, Hoepelman AIM. Wound infections in repatriated survivors of the tsunami disaster. Wounds. 2006;18(4):92-100.

17. Noordergraaf GJ, Bouman JH, van den Brink EJ, van de Pompe C, Savelkoul TJ. Development of computer-assisted patient control for use in the hospital setting during mass casualty incidents. Am J Emerg Med May. 1996;14(3):257-61.

18. Marres GMH, Vermetten E, van der Laan L, Mulder PGH, Leenen LPH. Disaster-related injuries and quality of life: a five year prospective follow-up in a non-residential sample after the 2004 Tsunami (Submitted-under review); 2010.

19. Marres GMH, Leenen LPH, Van der Slikke JW, Vermetten E. Use of a web portal for support and research after a disasteropportunities and lessons learned. Interact J Med Res (serial on the Internet). 2010; Aug(1). Available from http://knol.google. com/k/g-m-h-marres/use-of-a-web-portal-for-support-and/306jme $30 s 9 \mathrm{~s} 2 \mathrm{x} / 3$.

20. Grundmann H, Aanensen DM, van den Wijngaard CC, Spratt BG, Harmsen D, Friedrich AW. The European Staphylococcal Reference Laboratory Working Group. Geographic distribution of staphylococcus aureus causing invasive infections in Europe: A molecular-epidemiological analysis. PLoS Med. 2010;7(1): e1000215.

21. Winkelhagen J, Bijlsma TS, Bloemers FW, Heetveld MJ, Goslings JC. Airplane crash near schiphol airport 25 February 2009: Injuries and casualty distribution. Ned Tijdschr Geneeskd. 2009;154(4):A1064.

22. Baker SP, O'Neill B, Haddon W Jr, Long WB. The injury severity score: a method for describing patients with multiple injuries and evaluating emergency care. J Trauma. 1974;14(3):187-96.

23. Hirshberg A, Holcomb JB, Mattox KL. Hospital trauma care in multiple-casualty incidents: A critical view. Ann Emerg Med Jun. 2001;37(6):647-52. 